\title{
Liquid-Crystalline Curvature Electricity: The Bending Mode of MBBA
}

\author{
D. SCHMidT, M. SchaDT, and W. HeLfRich \\ Physics Department, F. Hoffmann-La Roche \& Co. Ltd., Basle, Switzerland \\ (Z. Naturforsch. 27 a, 277-280 [1972] ; received 23 November 1971)
}

\begin{abstract}
Measurements are reported which show that the room-temperature nematic MBBA can be deformed by means of curvature electricity if it is contained in a thin sandwich cell with the electric field parallel and the unique axis normal to the bounding walls. Meyer's coefficient $e_{33}$ was determined to be $4 \cdot 10^{-5} d y n e^{1 / 2}$ at room temperature; and its sign was found to be positive.
\end{abstract}

\section{Introduction}

It has recently been pointed out ${ }^{1}$ that the bending mode of curvature electricity $(=$ piezoelectricity) ${ }^{2}$ of nematic liquid orystals may be observable in a very simple way. An experiment of the considered kind had been reported by HAAs et al. ${ }^{3}$ who, however, did not interpret it. In the following, we describe similar measurements which are more complete and appear to prove that curvature electricity is, indeed, the acting mechanism.

The liquid crystal used in the earlier and present experiments was the room-temperature nematic MBBA ( $\mathrm{p}^{\prime}$-methoxybenzylidene- $\mathrm{p}, \mathrm{n}$-butylaniline). It was sandwiched between two parallel glass plates, the orientation being normal to the glass in the fieldless state. The curvature-electric effect in question consists in a slight bending of the orientation lines in an electric field $E$ parallel to the glass. With $\varphi(x)$ being the angle by which the alignment is tilted into the field direction and $x$ the coordinate normal to the glass, one has ${ }^{1,2}$

$$
\mathrm{d} \varphi / \mathrm{d} x=e_{33} E / K_{33}
$$

where $e_{33}$ is the curvature-electric coefficient and $K_{33}$ the elastic modulus, both for bend. Positive $\mathrm{d} \varphi / \mathrm{d} x$ means that field and induced bend relate to each other like arrow and bow. It is assumed here that the aligning force of the walls is weak enough not to influence $\mathrm{d} \varphi / \mathrm{d} x$. We also expect $|\varphi|$ to be equal on both bounding walls, thus being zero in the middle.

The refractive index $n$ for light of normal incidence travelling through the sandwich cell and polarized parallel to the field depends on the angle $\varphi$. It is given by

$$
\frac{1}{n}=\sqrt{\frac{1}{n_{\mathrm{e}}^{2}} \sin ^{2} \varphi+\frac{1}{n_{0}^{2}} \cos ^{2} \varphi}
$$

where $n_{0}$ and $n_{\mathrm{e}}$ are the "ordinary" and "extraordinary" refractive indices of the liquid crystal. For small angles $|\varphi| \ll \pi / 2$ one may use

$$
n-n_{\mathrm{o}}=\frac{1}{2} n_{\mathrm{o}}\left(1-\frac{n_{\mathrm{o}}^{2}}{n_{\mathrm{e}}^{2}}\right) \varphi^{2} .
$$

Accordingly, the optical path difference $\Delta l$ produced by the considered curvature-electric deformation of the orientation pattern is

$$
\Delta l=\left(\frac{\mathrm{d} \varphi}{\mathrm{d} x}\right)^{2} n_{\mathrm{o}}\left(1-\frac{n_{\mathrm{o}}^{2}}{n_{\mathrm{e}}^{2}}\right) \frac{d^{3}}{24} .
$$

The corresponding formula of Ref. ${ }^{1}$

$$
\Delta l=(\mathrm{d} \varphi / \mathrm{d} x)^{2}\left(n_{\mathrm{e}}-n_{\mathrm{o}}\right)\left(d^{3} / 12\right)
$$

is simpler, but valid only for $\left|\left(n_{\mathrm{o}} / n_{\mathrm{e}}\right)-1\right| \ll 1$, i. e. for weak optical anisotropy.

\section{Determination of $\left|e_{33}\right|$}

In most of the experiments, two parallel strips of aluminum foil were used as electrodes and spacers. Their thickness varied between 15 and $220 \mu$, the distance being about $2 \mathrm{~mm}$. The unique axis of MBBA was oriented perpendicular to the bounding microscope cover slides by means of a thin layer of lecithin. To put on the coating, the glass was dipped in a hot alcoholic lecithin solution. Upon applying a DC voltage to the electrodes, the sample appeared biaxial in light transmitted normally to the cell. The observation, either straightforward or conoscopic, was made under a polarizing microscope. The electrical birefringence was positive, i. e. the larger of the two apparent refractive indices was in the direction of the field. It was measured with an elliptical compensator $(\lambda / 10)$, for large

Reprint requests to Dr. W. HeLfrich, Abt. VI/Phy, F. Hoffmann-La Roche \& Co. Ltd., CH-4002 Basel, Switzerland. 


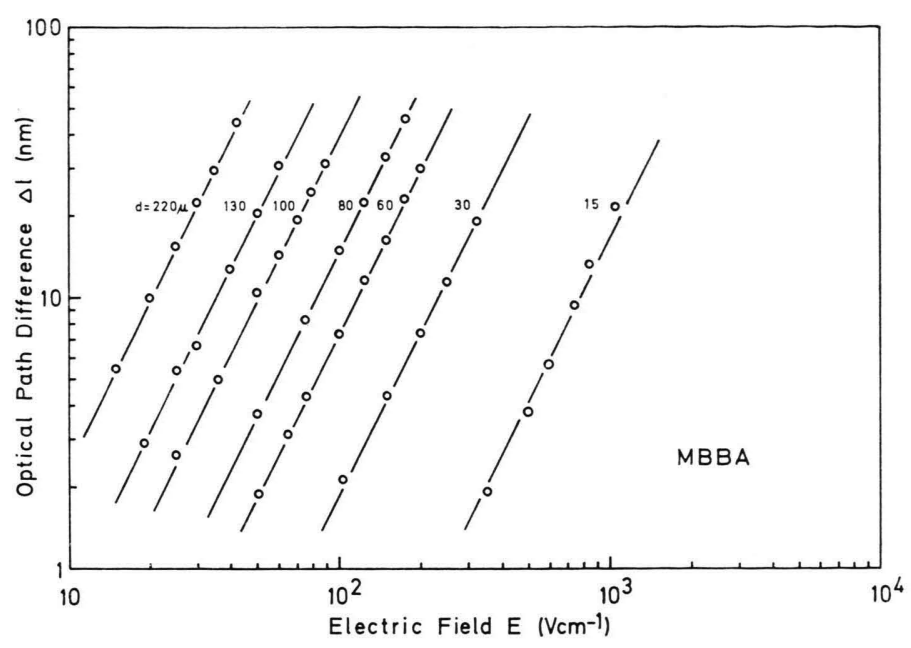

Fig. 1. Optical path difference versus electrical field strength for samples of varying thickness.

Fig. 2. Optical path difference as function of sample thickness at fixed electric field.

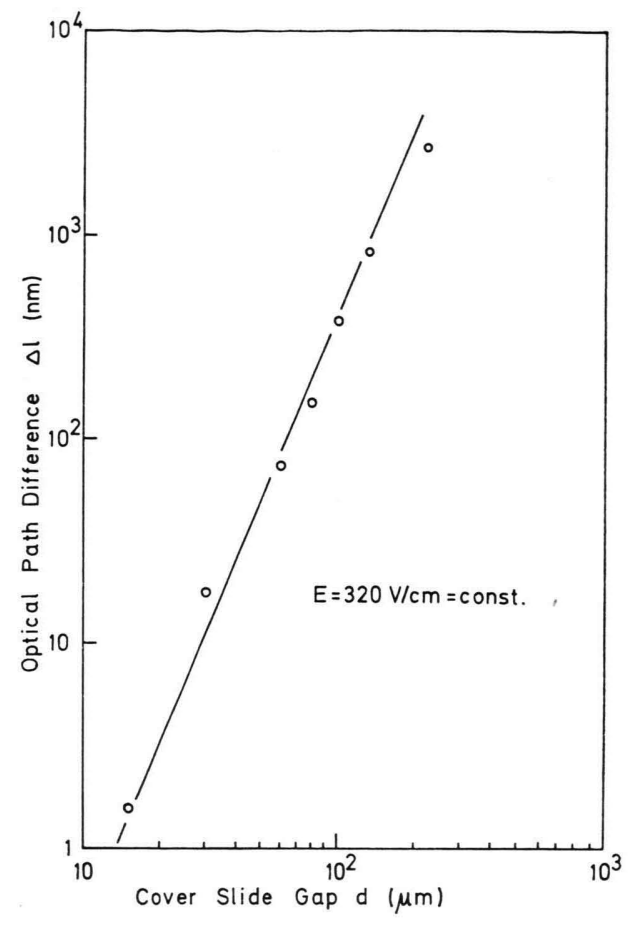

values a Bereck compensator $(4, \lambda)$ was used. All experiments were performed in green light $(54.1 \mathrm{~nm})$ and at room temperature. Careful observation showed the induced birefringence to be the same all over the sample (except immediately at the electrodes). Apparently, the electric field was uniform despite the thinness of the liquid crystal. This may be anticipated, as liquid crystals are usually impure, thus being weak electrolytes rather than insulators. All measurements were made at room temperature (ca. $\left.22{ }^{\circ} \mathrm{C}\right)$.

Figure 1 shows the measured optical path difference vs. electric field, the cell thickness being the parameter. Each function obeys a square law, as predicted by the curvature-electric model and suggested by the earlier experiment ${ }^{3}$. The thickness dependence of $\Delta l$ at constant field is plotted in Figure 2. The best fit for the data appears to be a cubic law (except, perhaps, for the uppermost point). This conforms with curvature electricity and indicates that the rigidity of wall alignment was negligible ${ }^{4}$.

The refractive indices of MBBA at $22{ }^{\circ} \mathrm{C}$ have been measured ${ }^{5}$; for $541 \mathrm{~nm}$ we deduce by interpolation

$$
n_{0}=1.557 \quad \text { and } \quad n_{\mathrm{e}}=1.792 .
$$

Inserting these in (2) and using Fig. 2, we derive

$$
|\mathrm{d} \varphi / \mathrm{d} x|=49 \mathrm{~cm}^{-1} \text { at } 320 \mathrm{~V} \mathrm{~cm}^{-1} \text {. }
$$

Substitution in (1) gives

$$
\left|e_{33} / K_{33}\right|=46 \mathrm{dyne}^{-1 / 2} \text {. }
$$

The clearing point of our MBBA was about $40{ }^{\circ} \mathrm{C}$. From unpublished data ${ }^{6}$ we take it that at $22{ }^{\circ} \mathrm{C}$ the elastic moduli should be

$$
K_{33}=8 \cdot 10^{-7} \text { dyne, }
$$

Finally we obtain

$$
\left|e_{33}\right|=3.7 \cdot 10^{-5} \mathrm{dyne}^{1 / 2} .
$$

The error may be up to $15 \%$, largely because of uncontrolled variations of the clearing point $\left(44{ }^{\circ} \mathrm{C}\right.$ in very pure samples, but only $40{ }^{\circ} \mathrm{C}$ in some of our measurements).

The slight deflection of the unique axis towards the direction of the field could not be caused by dielectric torques because the dielectric anisotropy of MBBA is negative $(\varepsilon \|<\varepsilon \perp)^{7}$. No birefringence was seen with $\mathrm{AC}(60 \mathrm{~Hz})$ instead of $\mathrm{DC}$, and a sudden reversal of the voltage made the birefringence slowly disappear and then reappear. These observations are further indications of curvature electricity. We also convinced ourselves that what 
we saw was not due to electrohydrodynamic realignment. Vortical flow was visible at elevated voltages, but all our data were taken below its apparent threshold. Theoretical arguments against vortical flow are the small voltages $(2 \mathrm{~V})$ down to which the measurements could be made, and the absence of a threshold for the birefringence.

\section{Determination of the Sign}

With a slightly different set-up, we have done additional experiments in order to determine the sign of $e_{33}$. We started from the notion that the curvature-electric bend should induce a weak electrohydrodynamic flow in a sufficiently conductive liquid crystal if the electrodes do not act as mechanical barriers. Accordingly, we used evaporated tin oxide strips of negligible thickness as electrodes. Mylar strips served as spacers; they were perpendicular to the electrodes and covered their ends. The field in the sample layer was limited to the area between the electrodes, as borne out by the electrooptic measurements. The layer extended farther and formed meniscuses at either end between the spacers. It was checked that the field-induced birefringence was the same, within experimental error, as with mechanically blocking electrodes.

The space charge density $\varrho$ associated with the curvature is given by ${ }^{8}$

$$
\varrho=-\frac{\varepsilon \| E}{4 \pi}\left(\frac{\varepsilon_{\perp}}{\varepsilon \|}-\frac{\sigma_{\perp}}{\sigma_{\|}}\right) \frac{\mathrm{d} \varphi}{\mathrm{d} x}
$$

where $\varepsilon$ and $\sigma$ are the dielectric constant and the conductivity, taken either parallel or perpendicular to the nematic axis. The average velocity $v$ of a liquid-crystalline layer in a uniform field $E$ is easily deduced to be

$$
v=\frac{1}{12} \cdot \frac{\varrho E d^{2}}{\eta_{1}}
$$

where $\eta_{1}$ is a viscosity coefficient ${ }^{8}$. Insertion of (1) in (3) and (3) in (4) yields the final formula for $v$. We note that $v$ has no voltage threshold and is proportional to $E^{3}$. Obviously, the flow changes sign with the applied voltage, being parallel to the field for $e_{33}<0$ and opposite for $e_{33}>0$.

We compute the velocity, taking known data ${ }^{7}$ for the electric properties $\left(\varepsilon \|=4.7, \varepsilon_{\perp} / \varepsilon_{\|}=1.13, \sigma_{\perp} / \sigma_{\|}\right.$ $=0.67)$. With $E=320 \mathrm{~V} \mathrm{~cm}^{-1}, d=100 \mu,\left|e_{33} / K_{33}\right|$
$=46 \mathrm{~cm}^{-1}$, and $\eta_{1}=1$ poise ${ }^{9}$ we obtain $v=6 \cdot 10^{-5}$ $\mathrm{cm} \mathrm{sec}^{-1}$. The experimentally observed motion of the meniscuses tended to agree within a factor of two with the theoretical value, if allowance was made for the friction in the fieldless part of the sample. However, a systematic study was not undertaken, partly because the flow was often held up by irregularities on the glass walls. The more important observation was the direction of flow. It was found to be opposite to the electric field, thus indicating

$$
e_{33}>0 \text {. }
$$

\section{Discussion}

Our result for $\left|e_{33} / K_{33}\right|$ is nearly an order of magnitude smaller than the value calculated ${ }^{1}$ from the work of HAAs et al. ${ }^{2}$. $e_{33}$ happens to equal the typical figure estimated on the basis of a molecular theory of curvature electricity ${ }^{10}$. A positive sign of $e_{33}$ would also agree with theoretical expectations, as may be seen from the structure of the MBBA molecule which is shown in Figure 3. The molecule

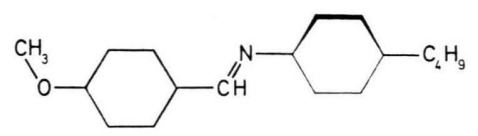

Fig. 3. The geometry of the MBBA molecule. (The twist of the right-hand benzene ring with respect to the rest of the molecule is indicated.)

carries two electric dipoles which are of the order of 1 debye. The should rotate more or less independently around the long molecular axis, thus resulting in two essentially independent contributions to $e_{33}$. One dipole is located at the oxygen atom and most likely gives a positive contribution to $e_{33}$ because it points into the bow formed by the oxygen with the methyl group on one side and the rest of the molecule on the other. The other dipole is attached to the link between the benzene rings, probably pointing from the nitrogen atom to the $\mathrm{CH}$ group. Its contribution may be comparatively weak and the sign appears difficult to predict, although a positive sign seems more likely to us, particularly if the two benzene rings are strongly rotated with respect to each other. A detailed calculation of $e_{33}$ from molecular properties seems premature in view of a lack of data. (E. g., the rotation angle between the benzene rings seems to be un- 
known. Also, it is not known whether the axes through the two benzene rings are exactly parallel.) Summing up, we think that we have obtained con-

1 W. Helfrich, Physics Letters 35 A, 393 [1971].

2 R. B. Meyer, Phys. Rev. Letters 22, 918 [1969].

3 W. Haas, J. Adams, and J. B. Flannery, Phys. Rev. Letters 25, 1326 [1970].

4 Deviations from the square law at large $d$ are discussed in Ref. ${ }^{1}$. Figure 2 together with Eq. (2) of Ref. ${ }^{1}$ yields for the rigidity of wall alignment $C<1 \cdot 10^{-4} \mathrm{erg} \mathrm{cm}^{-2}$ if we take for $K_{33}$ the value used in the present paper. clusive evidence for liquid-crystalline curvature electricity and a coefficient $e_{33}$ of reasonable sign and magnitude.

5 M. Brunet-Germain, C. R. Acad. Sci. Paris 271 B, 1075 [1970].

${ }^{6}$ I. Haller, R. B. Meyer, H. Gruler, and G. Meier, private communication.

7 D. Diguet, F. Rondelez, and G. Durand, C. R. Acad. Sci. Paris 271 B, 954 [1971].

8 W. Helfrich, J. Chem. Phys. 51, 4092 [1969].

9 C. GÄHWILLER, Physics Letters 36 A, 311 [1971].

10 W. Helfrich, Z. Naturforsch. 26 a, 833 [1971].

\title{
On the Migration of Ions in Alkali Nitrate-Silver Nitrate Melts
}

\author{
J. Richter and E. Amkreutz \\ Lehrstuhl für Physikalische Chemie II der Rheinisch-Westfälischen Technischen Hochschule Aachen \\ (Z. Naturforsch. 27 a, 280-287 [1972] ; received 5 October 1971)

\begin{abstract}
Stoichiometric transport numbers of the ion constituents of the molten salt mixtures
\end{abstract} \\ $\mathrm{NaNO}_{3}+\mathrm{AgNO}_{3}$ and $\mathrm{LiNO}_{3}+\mathrm{AgNO}_{3}$ (referred to the common anion) have been determined \\ by EMF measurements in a concentration cell with transference. The composition range is \\ $0.7 \leqq x \leqq 1.0$ at $240{ }^{\circ} \mathrm{C}$ to $0.1 \leqq x \leqq 1.0$ at $300{ }^{\circ} \mathrm{C}$ ( $x$ denotes the mole fraction of silver nitrate) \\ in the system $\mathrm{NaNO}_{3}+\mathrm{AgNO}_{3}$, and $0.1 \leqq x \leqq 1.0$ in the same temperature range in the system \\ $\mathrm{LiNO}_{3}+\mathrm{AgNO}_{3}$, the phase diagram defining the lower limit and the decomposition of the melt \\ the upper limit. In addition, ionic mobilities, conductivities, equivalent conductivities, and the \\ characteristic quantities of the idealized melts of the two systems have been calculated at $260{ }^{\circ} \mathrm{C}$ \\ and $290{ }^{\circ} \mathrm{C}$.
}

\section{Introduction}

In 1968 SINISTRI ${ }^{1}$ wrote: "Careful measurements of transport numbers of these systems $\left(\mathrm{NaNO}_{3}+\right.$ $\mathrm{AgNO}_{3}, \mathrm{KNO}_{3}+\mathrm{AgNO}_{3}$ ) would be highly desirable,...". At this time, measurements of these quantities by the Hittorf method existed for the system $\mathrm{NaNO}_{3}+\mathrm{AgNO}_{3}$ by Duke, LAITY, and Owens ${ }^{2}$ and for the system $\mathrm{KNO}_{3}+\mathrm{AgNO}_{3}$ by DuKE and Owens ${ }^{3}$. These measurements "are scanty and somewhat uncertain" (SINISTRI ${ }^{1}$ ). Since then the work of OKADA and KAWAMURA ${ }^{4}$ has been published; these authors determined the transport numbers for $\mathrm{KNO}_{3}+\mathrm{AgNO}_{3}$ at $300{ }^{\circ} \mathrm{C}$ by EMF measurements in a concentration cell with transference. The electrodes of this cell were two nitrate electrodes as developed by KetelaAr and DAmmers-DeKLeRK ${ }^{5}$. The transport numbers of Okada and Kawamura agree very well with those of Duke and Owens.

The purpose of this paper is the determination of the stoichiometric transport numbers of the ion constituents in the systems $\mathrm{NaNO}_{3}+\mathrm{AgNO}_{3}$ and
$\mathrm{LiNO}_{3}+\mathrm{AgNO}_{3}$ by EMF measurements in a concentration cell with transference, as functions of composition and temperature. The activity coefficients required for the analysis were also obtained in our laboratory ${ }^{6}$ from EMF measurements in a chemical cell containing a nitrate electrode of the type cited above ${ }^{5}$. Thus the uniformity of the experimental methods and of the possible errors are guaranteed. The transport numbers of the system $\mathrm{NaNO}_{3}+\mathrm{AgNO}_{3}$ determined here can be compared to those of Duke, LAITy, and Owens ${ }^{2}$ and of AzIZ and WETMORE ${ }^{7}$. The transport numbers of the system $\mathrm{LiNO}_{3}+\mathrm{AgNO}_{3}$ can be confronted with those of KAWAMURA and OKADA ${ }^{8}$ recently published.

The ionic mobilities and conductivities of the ion constituents and the equavilent conductivities of the two systems follow from the transport numbers and from the densities and electrical conductivities of the mixtures. The equivalent conductivities and the transport numbers of the idealized melts can be calculated from these ionic conductivities.

Reprint requests to Dr. J. Richter, Lehrstuhl für Physikalische Chemie II der RWTH Aachen, D-5100 Aachen, Templergraben 59 , West-Germany. 